\title{
Research on the Differentiation of Human Resource in Sports Industry
}

\author{
Xiaolong Weng \\ Xi'an International University; Xi'an 710077, China
}

\begin{abstract}
Keywords: sports industry; human resource; analysis and research.
\end{abstract}
\begin{abstract}
Sports industry is organized to satisfy people's demand for sports consuming, and it is regarded as the symbol of modern sports industry development. People have increasing promotion with demands for sports entertainment, but human resource setting of sports industry is not enough in general and lacks professional talents. So scientific and accurate forecast, analysis and human resource work organization are to realize good human resource supply and demand proportion so as to comprehensively face with the change of enterprises on developing strategies and clarify the emphasis of current human resource management work. This paper made the specific analysis with the current status of human resource management of sports industry and premises to achieve the supply and demand balance of human resource, and it made the differential analysis with enterprises human resources on the basis of knowing enterprise human resource management strategies and human resource balance. And it made corresponding analysis on suggestions and solutions aiming at existing problems so as to better develop service for human resource management work of sports industry and real development of enterprises.
\end{abstract}

\section{Introduction}

Human resource management is to explore the new possibility of enterprise management, and realize the standardized management of enterprise human cost and resources. But strengthening the compatibility of human resource management and enterprise strategy is more conducive to the overall development of modern enterprises, which better manifests the immense meaning of supply and demand balance of human resources. The system of sports industry is huge and the application of human resources is highly differential. So the differential analysis of human resources can make more rational allocation of human resources, having rather great influence on enhancing comprehensive application level of enterprise talents in real life and promote the harmonious development of sports industry. Meanwhile, human resources mean the overall coordination and management with corresponding enterprise capital, talents and things to a great extent. The imbalance of supply and demand will make the development of enterprise limited. Strengthening the analysis with differential management of human resources under this reality environment will be more easy to form strategic solutions and help the sports industry to realize comprehensive development of management work.

\section{Related Factors that Affect the Differential Configuration of Human Resources in Enterprises}

Human resource management is a complex and huge project, and sports industry has same characteristics. The allocation process of human resources is always the dynamic change of supply and demand. At the same time of realizing differential configuration, the reality of human resources should be considered. Finding the point to make the supply and demand balance of human resources in different departments is also the point to make resourced get optimal configuration. So reasonable economists long to realize the rational configuration with related resources in the optimal stage to find more profits. It is same for human resources, which means the factors affecting the balance of human resources mainly come from two aspects: external supply and internal demand.

\subsection{External Supply Factors}

Analysis of human resources demand tends to unfold with the center of "thing", and the analysis of human resources supply should be oriented with "people". Conditions of external labor force market 
determine the part of enterprise recruitment to a great degree. When the external labor force makes is tense, the quantity of external supply will reduce and human resource demand will be expanded to a certain degree. Decisive factors of external supply also include season, industry, human resource demand and so on. As for season, human resource supply in winter is less compared with Spring and Summer. As for the industry, human resource supply in ordinary industry is rather abundant, while the special industries (such as engineering technology and computer) have bigger difference in regional supply.

\subsection{Internal Demand Factors}

The demand for human resources shows a certain introversion, that is, the flow of human resources in the human resources market is mainly driven by the actual needs of enterprises. And the internal demand is more complex, which has a great relationship with the business status, development stage, salary level and strategic objectives. In terms of business conditions, good human resource demand is easier to be guaranteed in good performance, expansion of products and increase of market input, and the increase of human resources will be of great significance to the management of enterprises and the development of products. In addition, with the different stages of enterprise development, the demand for human resources is also different. This is because, with the different stages of enterprise development, the quantity of tasks and the actual needs of human resources are also different, which leads to the change of human resource demand. The change of demand mainly occurs in the growth and decline period of enterprises. In the period of enterprise growth, business is gradually expanding and business volume is also increasing. In addition, the organizational results of enterprises are becoming complicated. In this period, the demand for human resources of enterprises is also increasing, while in the declining period of the enterprise, the business of the enterprise is gradually decreasing, and the result of the organization of the enterprise tends to be concise. During the recession, the jobs and staff needed by enterprises are also decreasing. With the change of enterprise life cycle and organizational structure and business, the human resource demand of enterprises has also changed. At this time, enterprises will adopt a series of supply adjustment strategies to regulate the supply and demand of human resources. To achieve a balance between supply and demand, two conditions must be satisfied. The first is that enterprises can accurately determine the demand changes of human resources in enterprises. The second is that manpower adjustment strategy of enterprise can guarantee the demand of human resources for enterprises.

\section{Related Countermeasures to Realize the Human Resource Differentiation in Sports Industry}

\subsection{Optimize the Process of Talents Introduction and Realize Talents Distribution Differentiation}

Scientific, reasonable and standardized recruitment process can effectively carry out the recruitment process and achieve satisfactory results. On the other hand, if the enterprise does not have a scientific recruitment workflow, then the enterprise will waste human, physical and financial resources, even affecting the process of whole recruitment work. Therefore, the recruitment process should be optimized scientifically. The recruitment of sports industry enterprises is recommended in accordance with the following procedures. Firstly, making the job analysis. Refer to the performance report and operating conditions of previous year to assess whether the required personnel are required to be employed and the conditions of employment. Secondly, build a quality model to take the skills into consideration. Thirdly, conduct market personnel analysis, compare the position information with the current recruitment information of the market and check the talents employment conditions and employment criteria of large enterprises and other small and medium-sized enterprises for the same position. Fourthly, select the channel of recruitment and choose different channels according to the job characteristics. Fifthly, resume screening and selecting a matching resume. Sixthly, evaluation center technology, including psychological test, interview and other comprehensive assessment of the 
candidates. If necessary, successful assessment methods of some big enterprises can be referred. At last, comprehensively check employee's conditions.

\subsection{Complete Talents Recruitment Plan and Realize Talents Strategy Differentiation}

The sports industry should incorporate the development strategy and human resource recruitment plan into the recruitment mode. The plan of human resources should be carried out according to the strategic planning and development goals of the corresponding enterprises. The vital link of improving the recruitment planning is to meet the business strategy of the enterprise, which is the objective plan of the enterprise for its comprehensive development and planning. As is said above, the operational planning of modern sports industry is a continuous step to a higher level. Therefore, the key of talent differentiation in the process of leapfrog development is the planning of recruitment. To be specific:

1. Strengthen cooperation within the industry and focus on the overall development. The recruitment of sports industry often represents the complexity of personnel composition and the complicated management regulations. Therefore, in its development path toward internationalization, former recruitment management system and idea should be reformed and the coordination among different departments should be enhanced. Regard the development general situation as the strategic goal and avoid the desertion in thought or behavior.

2. Learn excellent recruitment planning system from international enterprises and implement strategic recruitment plan to make talents with different types have proper development space and then promote the overall development of the industry. Strategic planning system is the refraction of human resource management. So it has to be cut from multiple angles: learn advanced management experience of western sports enterprises, from the recruiter configuration to the management process, rules and regulations. It can be used to modify the trajectory of enterprise development, move towards higher goals and accelerate the effectiveness of strategic management.

In addition, for the human resources differential configuration of the sports industry, the first thing is that it should be based on the human resource planning and master the recruitment period, the number and type and talents quality through human resource planning. Recruitment planning should incorporate internal and external factors and should consider the following aspects: position arrangement, recruitment plan, time and cycle.

In addition, for external recruitment channels, the recruitment position and quantity should consider the suitable selection, the design and comb with the recruitment process, the assessment and prediction of recruitment market and the prediction with all fees produced by the recruitment and evaluation of the recruitment effect. We can make scientific and reasonable recruitment planning through the above content so as to achieve the human resources required by the enterprise and promote the rapid development and realization of the target.

\section{Carry Out Industry Manpower Resource Analysis and Realize Human Resource Demand Differentiation.}

The current situation analysis of human resources in sports industry is mainly to clarify the current problems of industrial development and the direction of future development. Such method can better find the different conditions of talents gap and abundance so as to improve the differential configuration of human resources demands. The analysis includes age structure, seniority structure, skill and work performance, etc. It also analyzes the turnover of personnel of one position or special department in recently one month, three months and six months, which can be used to understand the imbalance point and main demands of industry human resources demand and take appropriate methods to supplement those positions where there exists frequent turnover of personnel. It includes performance incentive, basic salary bottom line increase, working time standardization and the guidance of bring human resources to industry department with more distinct prospect.

In the actual management of human resources, the traditional human resource management often ignores itself as a whole in a sense, and the decentralized management mode has a great influence on 
the improvement of the overall efficiency of the work, which is not conducive to the management of the staff. Through the establishment of reasonable performance management, a good combination of performance and variable salary system can be used to motivate the employees effectively. The unified performance assessment also eliminates the influence of external factors such as private or dark box operation on the management of enterprise human resources, which enables employees to maximize the contribution value under the constraint of a unified performance system, resulting in the intensification effect of human resource management. It is of great significance for the future development of an enterprise. This intensive effect is reflected not only in the intensive degree of production efficiency, but also in the work attitude, which makes the corporate culture and economic benefits develop well together.

\section{Actively Carry Out School-Enterprise Cooperation and Configure the Talent System Differentially}

In general, sports industry talents should understand the uniqueness of sports and the means of operating management and market operation, which is the new trend equipped with current sports industry. And it can connect famous domestic sports colleges, for instance, Beijing Sports University and Shenyang Institute of Physical Education build school education launching system with multiple layers. The sports industry should develop rapidly and the talents quality is the top priority. Education development is a good way to improve the capital of sports industry from scratch. Therefore, it is necessary to highlight the actual demand of the sports industry for talents and improve the connection of education resources within and outside the industry to form the training model of theory plus practice. Use good masters to take the class and cultivate those students with hard sports quality and great enthusiasm with future development of the sports industry.

Acceleration the cultivation of professionals in the training process outside the school can help students better finish the digestion and absorption with theoretical knowledge and have more comprehensive understanding with professional teaching practice model of hotels, which is of great significance to promoting the comprehensive competitiveness of sports industry talents.

\section{Conclusion}

Human resources management of sports industry is an vital link in perfecting the sports industry management organization. Along with the advent of knowledge economy era, human resources has gradually become the core resources of realizing industry strategic goals. Strengthen human resources management and implement differential configuration of human resources is the important way to realize the sustainable development of enterprises. The construction of human resources management system should consider from the long-term development targets of enterprises, and it should connect human resource planning, training and compensation management module to constantly optimize and realize the goals of human resource management strategy together. In addition, the construction of enterprise human resources management system should be oriented with the actual development of sports industry, not copying other industry management systems. The development situation and personnel demand of each industry vary from each other, so there are certain differences in the way to manage the difference management of human resources. Therefore, in the process of sports industry development, recruitment management system should constantly be innovated and improved to improve the efficiency of differential configuration of human resources.

\section{Acknowledgements}

Scientific research project of Shaanxi provincial education department in 2017(17JK1092)

Project Name: the path of human resources supply in Shaanxi sports service industry. 


\section{References}

[1]. Guobin Lan. International Operation of Sports Industry and Strategic Human Resource Management[A]. The 4th National Youth Sports Science Conference Paper Abstract Compilation[C].2005

[2]. Jing Liu and Linlin Zhang. Thinking on the Current Situation of Chinese Sports Industry Management Talents Shortage[J]. Journal of Shenyang Institute of Physical Education,2004,23(4):501-503

[3]. Jun Yang. Study on the Current Situation of Sports Economy and Talents in China. [J]. Journal of Chengdu Institute of Physical Education,2006

[4]. Hanbin Wang and Yixiao Huo. The Confirmation and Process Design of Establishment Principle of Enterprise Human Resource Planning Model Library[J]. Journal of Harbin Commercial University (Social science edition),2012(01):99-104.

[5]. Zhiming Fu. Analysis on Enterprise Human Resource Management Mode and Its Transformation Trend - from the Perspective of Human Resources Supply and Demand Management[J]. Shandong Social Science,2010(12):65-68. 